\title{
Adenomyosis: from the sign to the diagnosis. Imaging, diagnostic pitfalls and differential diagnosis: a pictorial review
}

\section{Adenomiosi: dal segno alla diagnosi. Imaging, errori diagnostici e diagnostica differenziale: pictorial review}

\author{
A.L. Valentini • S. Speca • B. Gui • B.G. Soglia • M. Miccò • L. Bonomo \\ Dipartimento di Bioimmagini e Scienze Radiologiche, Istituto di Radiologia, Università Cattolica del Sacro Cuore di Roma, \\ L.go A. Gemelli 8, 00168 Rome, Italy \\ Correspondence to: A.L. Valentini, Tel.: +39-06-30154394, Fax: +39-06-35501928, e-mail: alvalentini@ rm.unicatt.it
}

Received: 23 November 2010 / Accepted: 17 January 2011 / Published online: 4 September 2011

(C) Springer-Verlag 2011

\section{Erratum to: La radiol med DOI 10.1007/s11547-011-0714-5}

The original version of this article, unfortunately, contained a mistake. The name of the fourth author should have been G. Soglia instead of B.G. Soglia

Nella versione originale di questo articolo era contenuto un refuso accidentale. Il nome del quarto autore avrebbe dovuto essere G. Soglia anziché B.G. Soglia. 